\section{Szent-Györgyi von Nagyrapolt, Albert}

\author{
A. M. Gressner ${ }^{1}$ und O. A. Gressner ${ }^{2}$ \\ ${ }^{1}$ Labor Dr. Wisplinghoff Berlin, Berlin, Deutschland \\ ${ }^{2}$ Labor Dr. Wisplinghoff Köln, Köln, Deutschland
}

Lebensdaten Ungarischer Mediziner, geboren am 16. September 1893 in Budapest, gestorben am 22. Oktober 1986 in Woods Hole, Massachusetts.

Verdienste Studium der Humanmedizin ab 1911 in Budapest mit anschließender wissenschaftlicher Tätigkeit in Phar- makologie, Physiologie und Biochemie an verschiedenen europäischen (Prag, Berlin, Hamburg, Leiden, Groningen, Szeged, Budapest u. a.) und amerikanischen Universitäten (Cambridge, Rochester). Seine wissenschaftlichen Verdienste liegen besonders in der Erforschung biologischer Verbrennungsprozesse, Bioenergetik, Ascorbinsäure und $>$ Vitamin C sowie > Fumarsäure und Flavine. Szent-Györgyi von Nagyrapolt erhielt im Jahr 1937 den Nobelpreis für Medizin. Er stellte die Identität von Vitamin C und Ascorbinsäure fest und erkannte ihre antioxidative und Skorbutverhindernde Wirkung. 